\section{Phenanthrene Sorption to Soil Humic Acid and Different Humin Fractions}

BEI WEN, $*,+$ JING-JING ZHANG, ${ }^{\dagger}$

SHU-ZHEN ZHANG, ${ }^{\dagger}$ XIAO-QUAN SHAN, ${ }^{*},{ }^{\dagger}$

SHAHAMAT U. KHAN, ${ }^{*}$ AND

B A O S HAN XING ${ }^{\S}$

State Key Laboratory of Environmental Chemistry and

Ecotoxicology, Research Center for Eco-Environmental Science, Chinese Academy of Sciences, P. O. Box 2871, Beijing 100085,

China, Department of Chemistry and Biochemistry, MSN 3E2, George Mason University, 4400 University Drive, Fairfax, Virginia 22030-4444, and Department of Plant and Soil Science, University of Massachusetts, Stockbridge Hall, 80 Campus Center Way, Amherst, Massachusetts 01003-9246

This study was undertaken to provide an insight into the effect of heterogeneous soil organic matter (SOM) on the sorption of phenanthrene. Humic acid (HA) and humin were extracted from a peat soil. Humin was further fractionated into bound-humic acid (BHA), lipid, and insoluble residue (IR) fractions. Heterogeneous natures of these fractions were characterized by elemental analysis, ultraviolet-visible spectroscopy, Fourier transform infrared spectroscopy, and solid-state ${ }^{13} \mathrm{C}$ NMR. Aliphaticity of the fractions followed the order lipid $>B H A>H A>I R$, while the polarity order was IR $>$ BHA $>$ HA > lipid. Sorption of phenanthrene on these fractions fitted the Freundlich equation, suggesting that phenanthrene sorption isotherms of lipid were almost linear $(N=0.993)$, while those of $\mathrm{HA}, \mathrm{BHA}$, and IR were nonlinear, with $N$ values ranging from 0.723 to 0.910 . The $N$ values followed the order lipid $>\mathrm{HA}>\mathrm{BHA}>\mathrm{IR}$ and were significantly correlated inversely with their polarities $(p<0.05)$. Organic carbon-normalized sorption coefficients ( $\left.K_{\mathrm{FOC}}\right)$ were independent of aliphatic or aromatic contents of the SOM fractions. The results suggested that SOM, especially for the humin fractions, was highly heterogeneous in terms of elemental composition, structure, and polarity. Such heterogeneity was considered to be responsible for the nonlinear sorption of phenanthrene.

\section{Introduction}

Soil organic matter (SOM) is considered as the most important factor controlling fate and transport of hydrophobic organic contaminants (HOCs) in soils and sediments. Over the past decade, the hypothesis that SOM behaves strictly as a phase partitioning medium based on an assumption of homogeneous SOM has been controversial. Mounting evidence against partitioning are varying organic carbon normalized sorption capacities and isothermal sorption nonlinearity, very slow rates of sorption and desorption, sorption-desorption hysteresis, and solute-solute competition (1-4).

* Corresponding author phone: + 86-10-62923560; fax: + 8610-62923563; e-mail: bwen@rcees.ac.cn; xiaoquan@rcees.ac.cn.

+ Chinese Academy of Sciences.

‡ George Mason University.

$\S$ University of Massachusetts.
A great deal of evidence indicates that the sorption nonlinearity relates primarily to the heterogeneity of SOM $(1-3)$. Weber et al. (1) introduced the concept of multiple discrete reactive mineral and SOM domains to explain the nonlinear behavior of HOC sorption. Soils and sediments can be treated as heterogeneous combinations of active organic and inorganic components. Each component has its own sorption energy and sorptive property and exhibits either a nonlinear or linear sorption. The overall sorption isotherm is the sum of the sorption isotherms of the active components. These authors first hypothesized that there are two types of physically and chemically heterogeneous SOM, "soft" and "hard" carbons. Sorption of HOCs into the soft carbon SOM follows a nearly linear partitioning process, whereas sorption on the hard carbon SOM exhibits both adsorption and absorption or partitioning (1). A similar concept was also proposed independently by Xing and Pignatello (4). Total sorption is described as the sum of partitioning and adsorption domains, which are analogous to rubbery and glassy polymers. Glassy domains are related to nonlinear sorption and sorption-desorption hysteresis, whereas rubbery domains are proposed to account for linear sorption. Identification and characterization of sorption domains in SOM has been extensively performed. The high-surface-area carbonaceous materials (HSACM), such as coal, charcoal, coke, kerogen, black carbon, and soot have been demonstrated to be important sources of sorption nonlinearity and desorption hysteresis (5). However, even humic acid, widely regarded as a partitioning phase, can exhibit dual partitioning-adsorption processes at temperatures below its glass transition temperature (6).

SOM has diverse chemical compositions, structures, and conformations that depend on the origin and age of its source materials. Traditionally, SOM has been divided into three operationally defined fractions according to their saponifiable properties: fulvic acid (FA), humic acid (HA), and humin. The heterogeneous natures of fulvic and humic acids have been extensively characterized (3). The impacts of the physical and geochemical characteristics of HA on the sorption of HOCs have been studied $(7,8)$. Though nonlinear sorption of HOCs to HA was revealed, and was ascribed to the heterogeneity of HA, the role of HA in the nonlinear sorption is still a matter of debate. On the contrary, nonlinear sorption on humin fraction was well accepted (9).

Although the importance of humin for the sorption nonlinearity has been highlighted, few investigations have further fractionated humin into different fractions due to its intractable nature. Thus the physical and chemical nature of humin along with its sorption mechanisms for HOCs is the least understood among the three humic fractions. Rice and MacCarthy (10) reported a novel variation of the traditional alkaline-extraction isolation technique involving partitioning humin between an aqueous phase at varying $\mathrm{pH}$ and methylisobutylketone (MIBK). This method allows the isolation of not only humin from FA and HA, but also the components of humin from each other. Humin is experimentally fractionated into bound humic acid (BHA), lipid and insoluble residue (IR). Since the introduction of the MIBK method, several papers dealing with the characteristics of different humin fractions and distribution of HOCs in these fractions have been published (11-15). However, very little information is available on sorption behaviors of HOCs to each humin fraction, and the relevant sorption mechanisms are poorly understood.

The aims of the present study were (1) to investigate the structure and composition of HA and individual BHA, lipid, 
and IR fractions extracted from a peat soil with the MIBK method; (2) to examine the sorption of phenanthrene, a model HOC compound, on these SOM fractions; and (3) to explore the effects of structural or compositional heterogeneities of these SOM fractions on the sorption of phenanthrene.

\section{Materials and Methods}

Reagents. Reference standard of phenanthrene of spectrophotometric grade $(>98 \%)$ was purchased from Aldrich Chemical Co., Inc, and directly used without further purification. Phenanthrene was selected as a solute probe because it is commonly found in contaminated aquatic and subsurface environments, and it has been often used in many environmental studies. The aqueous solubility of phenanthrene at $22^{\circ} \mathrm{C}$ is $1.120 \mathrm{mg} / \mathrm{L}$, and $\log K_{\mathrm{o} w}$ is 4.45 (16). All the organic solvents used in this study were commercially available. Methanol (Mallinckrodt, U.S.) and methylisobutylketone (MIBK, Merck, U.S.) were of pesticide grade.

A primary phenanthrene stock solution at $1.0 \mathrm{mg} / \mathrm{mL}$ was prepared by dissolving an appropriate amount of the chemical in methanol. It was diluted with methanol to make a series of stock solutions at various phenanthrene concentrations. All stock solutions were stored at $4{ }^{\circ} \mathrm{C}$ in glass bottles sealed with Teflon-lined tops. A desired volume of stock solution was mixed with a background solution $(0.01 \mathrm{~mol} / \mathrm{L}$ $\mathrm{CaCl}_{2}$ and $200 \mathrm{mg} / \mathrm{L} \mathrm{NaN}_{3}, \mathrm{pH}$ 4) in a volumetric flask to make an initial aqueous solution for sorption experiments. Ten levels of initial solutions ranging from 20 to $1000 \mu \mathrm{g} / \mathrm{L}$ were prepared. The volumetric fraction of methanol in each initial solution was less than $0.2 \%$. At this level methanol had no measurable cosolvent effect on the sorption of organic pollutants (17).

Isolation of HA and Humin Fractions. A commercial peat soil was purchased from Jilin Changbai Mountain Peat Moss Development Co., Ltd. (Changchun, Jilin Province, China). Its organic matter content determined by the Walkley-Black procedure (18) was $46.5 \%$, and the $\mathrm{pH}$ value measured at peat soil to $0.01 \mathrm{~mol} / \mathrm{L} \mathrm{CaCl}_{2}$ solution ratio of $1: 1(\mathrm{w} / \mathrm{v})$ was 6.6.

HA and humin were isolated from peat soil according to the International Humic Substances Society (IHSS) procedure published on January 25, 1985. Humin was further fractionated according to MIBK procedure (10) (Supporting Information, Figure S1).

Characterization of HA and Individual Humin Fractions. The HA, BHA, lipid and IR fractions were characterized using standard methods. The elemental compositions (C, H, N, S) were analyzed with a high-temperature combustion method (PE 2400 SERIES II analyzer, Pekin-Elmer, Inc.). Oxygen content was calculated by the mass difference. Ash content was determined by combustion of SOM fractions at $800{ }^{\circ} \mathrm{C}$ for $4 \mathrm{~h}$. The ratios of absorbance at 465 and $665 \mathrm{~nm}\left(\mathrm{E}_{4} / \mathrm{E}_{6}\right)$ of $\mathrm{HA}$ and BHA were determined from aqueous solutions at pH 8 containing $0.05 \%$ (W/V) of HA or BHA by Agilent 8453 UV-visible spectrometer (U.S.). The $\mathrm{pH}$ was adjusted with dilute $\mathrm{NaOH}$ and $\mathrm{HCl}$ solution.

Infrared spectra were obtained on a NEXUS 670 Fourier transform infrared (FTIR) spectroscopy (Thermo Nicolet Company, Madison, Wisconsin) equipped with deuterated triglycine (DTGS) and mercury-cadmium-telluride (MCT) detectors (Thermo Nicolet Company), a KBr beam splitter (Thermo Nicolet Company) and a sample bench purged with dry air. The resolution of Fourier transform infrared (FTIR) spectroscopy was $2.0 \mathrm{~cm}^{-1}$, and a total of 64 scans were collected for each spectrum. The samples prepared from the soil suspensions were filtered with a $0.45 \mu \mathrm{m}$ hydrophilic polyethersulfone membrane and freeze-dried overnight. The Fourier transform infrared (FTIR) spectroscopy for pellets of a mixture of $5.0 \mathrm{mg}$ of SOM fractions and $100 \mathrm{mg}$ of dried $\mathrm{KBr}$ pressed under reduced pressure were recorded.

The HA, BHA, lipid, and IR fractions were also subjected to ${ }^{13} \mathrm{C}$ NMR analysis to obtain their chemical group distribution. All samples were treated with dilute hydrofluoric and hydrochloric acids to reduce ash and paramagnetic contents. Solid-state ${ }^{13} \mathrm{C}$ NMR data were acquired using a crosspolarization and magic angle spinning (CPMAS) on a 300MHz NMR spectrometer (Varian, San Francisco). Spectra were acquired at a frequency of $75 \mathrm{MHz}$ for ${ }^{13} \mathrm{C}$ MAS spinning rate of $13 \mathrm{kHz}$, contact time of $2 \mathrm{~ms}, 4 \mathrm{~s}$ recycle delay. The number of scans was from 5000 to 10000 per sample.

Sorption Experiments. A batch technique with $10.0 \mathrm{mg}$ sorbent was utilized for all sorption experiments in this study. A mixture of $0.01 \mathrm{M} \mathrm{CaCl}_{2}$ and $200 \mathrm{mg} / \mathrm{L} \mathrm{NaN}_{3}$ (pH 4.0) was used as background solution in all sorption experiments in order to prevent any potential dissolution of HA or BHA in solution and biological degradation of phenanthrene without causing significant changes in the organic matter chemistry. In brief, 40-mL glass tubes with Teflon-lined caps were used as batch reactors. The bottles were filled to minimal headspace with phenanthrene solution $(42.4 \mathrm{~mL})$ and sealed. According to our preliminary sorption rate and equilibrium studies, an equilibration time of $7 \mathrm{~d}$ was used. Our preliminary tests indicated that apparent equilibrium was reached before 3 days for HA, BHA, and lipid fractions and 6 days for IR fraction (Supporting Information, Figure S2). The initial aqueous-phase phenanthrene concentrations $\left(C_{0}\right.$ in $\left.\mathrm{mg} / \mathrm{L}\right)$ were selected to yield a set of isotherm data for each sample that distributed evenly on a log-log scale plot and to span approximately 2 orders of magnitude in aqueous-phase solute equilibrium concentrations $\left(C_{\mathrm{e}}\right.$ in $\left.\mathrm{mg} / \mathrm{L}\right)$. The amount of sample in each tube corresponded to a sample-to-solution ratio that would result in $30-85 \%$ uptake of phenanthrene. Reactors filled with sorbent and initial aqueous solution were mixed completely by shaking at $125 \mathrm{rpm}$ at room temperature $\left(21 \pm 2{ }^{\circ} \mathrm{C}\right)$ for 7 days. After centrifugation at $1667 \mathrm{~g}$ for 20 min, phenanthrene in the supernatant solution was determined by high performance liquid chromatograph (HPLC).

Control reactors prepared similarly but no sorbent were run simultaneously for assessing loss of solutes to reactor during sorption test. Results of triplicate sorption at each $C_{0}$ level showed that the average solution-phase concentrations of each solute were consistently within $98-102 \%$ of the respective initial concentration of the same solution, thereby indicating that microbial degradation and volatilization during the sorption and the uptake to the glass walls were negligible. Hence, no correction was made during sorption experiments.

The sorption data measured for phenanthrene on the HA, BHA, lipid, and IR fractions fitted the Freundlich isotherm model:

$$
\log S=\log K_{\mathrm{F}}+N \log C_{\mathrm{e}}
$$

Where $S$ is the solid-phase concentration $(\mathrm{mg} / \mathrm{kg})$ and $C_{\mathrm{e}}$ is the liquid-phase equilibrium concentration $(\mathrm{mg} / \mathrm{L})$. The parameter $K_{\mathrm{F}}$ is the sorption capacity coefficient $[(\mathrm{mg} / \mathrm{kg}) /$ $\left.(\mathrm{mg} / \mathrm{L})^{N}\right]$ and $N$ (dimensionless) is the isotherm nonlinearity, which was calculated by linear regression of log-transformed data using the Origin version 6.0 at $95 \%$ confidence.

Determination of Phenanthrene. The concentrations of phenanthrene were determined using a reversed-phase HPLC (C18 column, $4.6 \mathrm{~mm} \times 25 \mathrm{~cm}$, Agilent Technologies, Wilmington, U.S.) with a diode array detector for concentrations ranging from 50 to $1000 \mu \mathrm{g} / \mathrm{L}$ and a fluorescence detector for concentrations from approximately 0.5 to $50 \mu \mathrm{g} / \mathrm{L}$. Isocratic elution was performed at a flow rate of $0.9 \mathrm{~mL} / \mathrm{min}$ using the mobile phase methanol: water (90:10). Under such HPLC conditions phenanthrene showed a single peak 


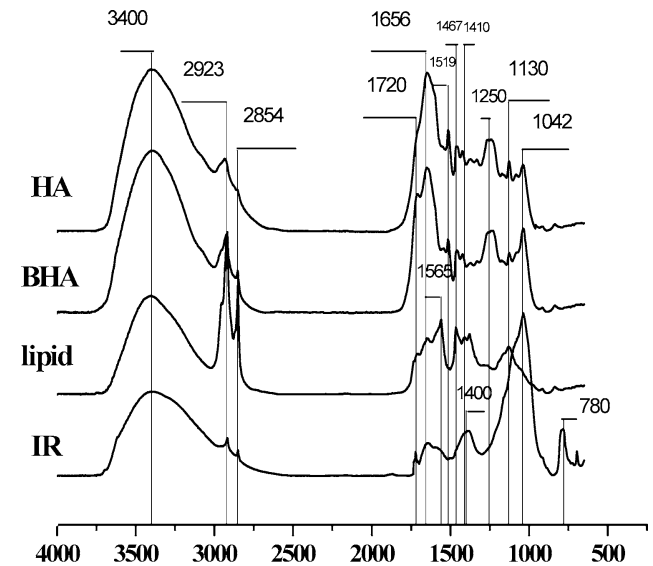

Wavenumber $\left(\mathrm{cm}^{-1}\right)$

FIGURE 1. Infrared spectra of SOM fractions.

at a retention time of $7.9 \mathrm{~min}$. The solid-phase sorbate phenanthrene concentration was calculated for each reactor from a solute mass balance between the two phases.

\section{Results and Discussion}

Elemental Compositions of SOM Fractions. Elemental compositions of four SOM fractions extracted from a peat soil are shown in Supporting Information Table S1. Ash contents were very low for the HA, BHA, and lipid fractions. The IR fraction had relatively high ash content although it had been previously treated with $\mathrm{HF}$ and $\mathrm{HCl}$. The $\mathrm{H} / \mathrm{C}$ ratios followed the order IR $<\mathrm{HA}<\mathrm{BHA}<$ lipid. A higher $\mathrm{H} / \mathrm{C}$ ratio may suggest a higher degree of aliphaticity (7). The polarity $[(\mathrm{N}+\mathrm{O}) / \mathrm{C}]$ was found to be the highest for the IR fraction, and the lowest for the lipid fraction. The BHA fraction had higher polarity than HA, which was consistent with the finding of Rice and MacCarthy (13).

Analyses of Fourier Transform Infrared (FTIR) Spectra. Differences in the Fourier transform infrared spectra among the HA, BHA, lipid, and IR fractions were observed (Figure 1). For all fractions, the broad band observed at about 3400 $\mathrm{cm}^{-1}$ is assigned to the stretching vibration of hydroxyl groups. The absorbance at 2923 and $2854 \mathrm{~cm}^{-1}$ results from aliphatic or alicyclic $\mathrm{C}-\mathrm{H}$ stretching. The band of 1720 and $1656 \mathrm{~cm}^{-1}$ is due to the $\mathrm{C}=\mathrm{O}$ stretching. The $1410-1467 \mathrm{~cm}^{-1}$ in the lipid fraction is the deformation of aliphatic $\mathrm{C}-\mathrm{H}$ (19). The absorbance at $1130 \mathrm{~cm}^{-1}$ is $\mathrm{C}-\mathrm{OH}$ stretching of aliphatic $\mathrm{O}-\mathrm{H}$. The peak that appears at $1042 \mathrm{~cm}^{-1}$ represents $\mathrm{C}-\mathrm{O}$ stretching of polysaccharides or polysaccharide-like substances. The absorbance at $1250 \mathrm{~cm}^{-1}$ is the $\mathrm{C}-\mathrm{H}$ stretching and $\mathrm{OH}$ deformation of $\mathrm{COOH}$ or $\mathrm{C}-\mathrm{O}$ stretching of aryl esters. The various bands between 800 and $600 \mathrm{~cm}^{-1}$ are likely from the mineral matrix.

The differences in FTIR spectra among the HA, BHA, lipid, and IR fractions represent their different physicochemical and structural nature. The absorbance at 2923 and 2854, $1410-1467$, and $1130 \mathrm{~cm}^{-1}$ in the lipid fraction are relatively large, implying the aliphatic nature of the lipid fraction (20). For the IR fraction, the absorbance at $1400 \mathrm{~cm}^{-1}$ is attributed to $\mathrm{C}-\mathrm{H}$ in plane deformation with the aromatic ring stretching (21), and the broad intensive band in the 1200$970 \mathrm{~cm}^{-1}$ range is due to both inorganic and organic compounds, $\mathrm{SiO}_{2}$, sulfates, silicates, and phosphates as well as carbohydrates, organic phosphates, and organosilicon compounds (vibrations of $\mathrm{C}-\mathrm{O}, \mathrm{C}-\mathrm{O}-\mathrm{C}, \mathrm{Si}-\mathrm{CHx}$, and $\mathrm{P}-\mathrm{O}-\mathrm{C}$ groups). All these absorption revealed the mineralbound organic matter characteristics of IR fractions. The absorption at $1519 \mathrm{~cm}^{-1}$ in HA and BHA may attribute to the $\mathrm{N}-\mathrm{H}$ deformation, $\mathrm{C}=\mathrm{N}$ stretching or aromatic $\mathrm{C}=\mathrm{C}$ stretch-

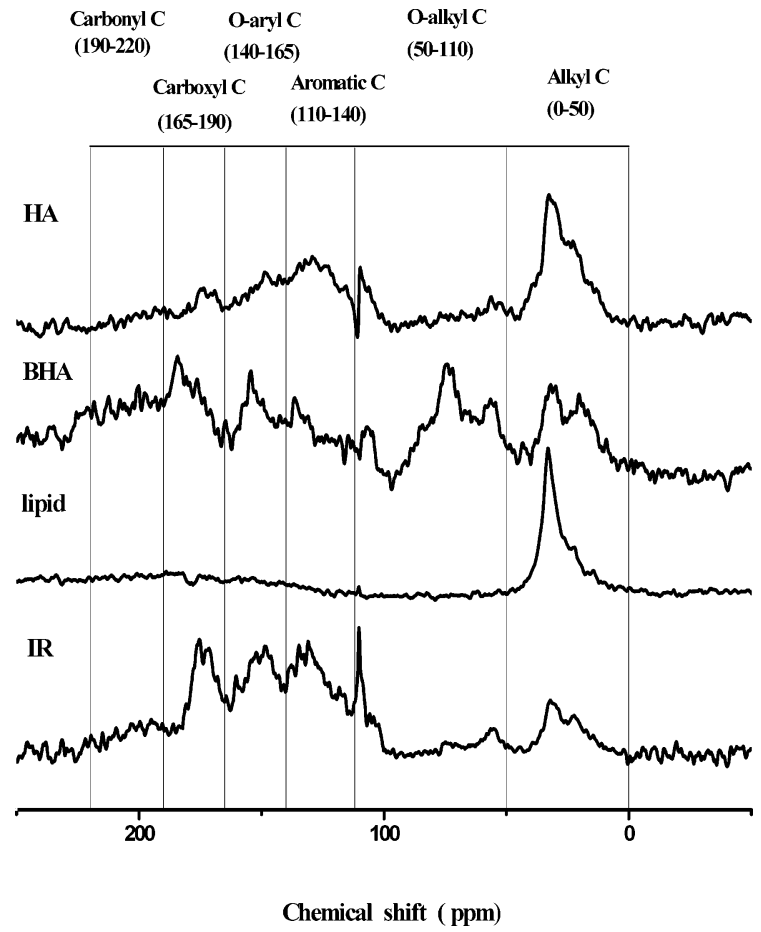

FIGURE 2. CPMAS ${ }^{13} \mathrm{C}$ NMR spectra of SOM fractions.

ing (19). The HA and BHA fractions had quite similar spectra, but the BHA fraction had relatively higher absorbance at 2923 and $2854 \mathrm{~cm}^{-1}$, as well as at $1720 \mathrm{~cm}^{-1}$, which showed its higher aliphatic and polar contents.

${ }^{13} \mathrm{C}$ NMR Spectra of SOM Fractions. The ${ }^{13} \mathrm{C} C \mathrm{CPMAS} N \mathrm{NR}$ spectra and integration results of the HA, BHA, lipid, and IR fractions are shown in Figure 2 and Supporting Information Table S2. The spectra were integrated as follows: alkyl-C (0-50 ppm); O-alkyl-C (predominantly carbohydrates, 50110 ppm); aromatic-C (110-165 ppm); carboxylic- and amide-C (165-190 ppm); and ketonic/aldehyde-C (190-220 $\mathrm{ppm}$ ). The percentage of polar organic carbon (POC) was calculated as the combined fractions of the 50-110 and 140$220 \mathrm{ppm}$ regions of the NMR spectra (22).

There are differences in carbon-type distributions among these SOM fractions. The lipid fraction had the highest alkyl-C content, while the IR had the highest aromatic-C content. Compared with HA fraction, the BHA fraction had more aliphatic carbon content. These observations are consistent with those reported by Rice and MacCarthy (13) including their elemental compositions (Table S1, Supporting Information). Overall, the aliphatic carbon content increased in the order IR $<\mathrm{HA}<\mathrm{BHA}<$ lipid, which was consistent with the increase in $\mathrm{H} / \mathrm{C}$ ratio. Xie et al. (14) found that the $\mathrm{O}$-alkyl-C were essentially absent from the BHA spectra when compared with those of HA. Rice (15) observed that in a peat BHA, the O-alkyl-C content was higher than that of $\mathrm{HA}$, while in soil BHA, the O-alkyl-C content was lower than that of HA. The wide range of resonances from 110 to $195 \mathrm{ppm}$ in the IR suggested that both aromatic and carboxyl carbons were present in this fraction. The POC of the BHA fraction was higher than that of HA. The POC was in the order IR > BHA $>$ HA > lipid, which was in line with the results of elemental composition and FTIR spectra analyses.

Polarities of SOM Fractions. Measurement of polarity of soil or geologic materials is very important because it plays an important role in the sorption behavior of organic pollutants $(7,23,24)$. The term polarity index as mass ratio $[(\mathrm{O}+\mathrm{N}) / \mathrm{C}]$ was proposed by Rutherford et al. (23). However, this definition was thought to ignore the polarity that may arise from configuration and structure. Thus, the POC 
TABLE 1. Freundlich Isotherm Parameters and Concentration-dependent Organic Carbon-Normalized Sorption Coefficient $K_{0 c}$ for the Soil Organic Matter Fractions

\begin{tabular}{|c|c|c|c|c|c|c|c|c|c|}
\hline \multirow[b]{2}{*}{ sorbents } & \multirow[b]{2}{*}{$\boldsymbol{N}$} & \multirow[b]{2}{*}{$K_{\mathrm{F}}^{b}$} & \multirow[b]{2}{*}{$F_{0 c}{ }^{a}$} & \multirow[b]{2}{*}{$K_{\mathrm{FOc}}{ }^{b}$} & \multirow[b]{2}{*}{$r^{2}$} & \multirow[b]{2}{*}{$n^{e}$} & \multicolumn{3}{|c|}{ concentration-dependent $K_{0 c^{c}}$} \\
\hline & & & & & & & $\begin{array}{c}\mathrm{Ce}=0.005 \\
(\mathrm{mg} / \mathrm{L})\end{array}$ & $\begin{array}{c}\mathrm{Ce}=0.05 \\
(\mathrm{mg} / \mathrm{L})\end{array}$ & $\begin{array}{c}\mathrm{Ce}=0.5 \\
(\mathrm{mg} / \mathrm{L})\end{array}$ \\
\hline HA & $0.910(0.003)^{d}$ & $6290(14.05)^{d}$ & 0.559 & 11298 & 0.999 & 30 & 18109 & 14726 & 11976 \\
\hline $\mathrm{BHA}$ & $0.868(0.012)$ & $5419(3.69)$ & 0.562 & 9679 & 0.999 & 30 & 19428 & 14326 & 10564 \\
\hline lipid & $0.993(0.007)$ & $29888(29.2)$ & 0.576 & 52158 & 0.999 & 30 & 54088 & 53223 & 52373 \\
\hline IR & $0.723(0.008)$ & $5288(13.56)$ & 0.335 & 15848 & 0.993 & 30 & 68764 & 36337 & 19202 \\
\hline
\end{tabular}

calculated from ${ }^{13} \mathrm{C} \mathrm{NMR}$ was recommended $(7,24)$. In this study, the polarity index of $(\mathrm{N}+\mathrm{O}) / \mathrm{C}$ was found to correlate well with POC from ${ }^{13} \mathrm{C}$ NMR spectra $(p<0.05)$ (Supporting Information, Figure S3). The polarity and proportion of aromatic carbon in soils were found to be strongly correlated inversely by Xing et al. (25), but such phenomenon was not found in this study (data not shown). Instead, negative correlation was found between polarity index and the alkyl carbon contents in organic sorbents though only four SOM fractions were tested $\left(R^{2}=0.996\right)$ (Supporting Information, Figure S4), which is in accordance with the results of Chen et al. (20). This suggests that alkyl carbon content and polarity of the organic sorbents as described in this study are two interactive parameters, which reflect the structure of sorbents and may affect the sorption of organic pollutants.

Sorption Isotherms. Phenanthrene sorption isotherms for the HA, BHA, lipid, and IR fractions fit eq 1. well, with the correlation coefficients higher than 0.99 (Supporting Information, Figure S5, Table 1). The sorption capacity coefficients $\left(K_{\mathrm{F}}\right)$ were calculated from the slope of the linear portion of the isotherms. The organic carbon-normalized sorption coefficient $\left(K_{\mathrm{FOC}}\right.$ ) was then calculated by dividing $K_{\mathrm{F}}$ values by the respective fraction of organic carbon $\left(F_{\mathrm{OC}}\right)$ in the sample. The concentration dependent $K_{\mathrm{OC}}(\mathrm{L} / \mathrm{kg}$ oc) was calculated based on the following equation:

$$
K_{\mathrm{OC}}=K_{\mathrm{FOC}} C_{\mathrm{e}}^{N-1}
$$

The values of $K_{\mathrm{OC}}$ can be used to denote directly the sorption characteristics of heterogeneous sorbents. The resulting model parameters, along with their standard errors $(\sigma)$, and the calculated $K_{\mathrm{OC}}$ values at $\mathrm{Ce}=0.005,0.05$, and $0.5 \mathrm{mg} / \mathrm{L}$ are presented in Table 1.

The Freundlich equation parameters suggested that the phenanthrene sorption isotherm of lipid was practically linear, with the $N$ value of 0.993 . While, sorption of phenanthrene onto the HA, BHA, and IR fractions was nonlinear, with $N$ values ranging from 0.723 to 0.910 . The $K_{\text {FOC }}$ values ranged from 9679 for BHA to 52158 for lipid. The sorption isotherm of phenanthrene on humin was also investigated. The $K_{\mathrm{FOC}}$ value of humin was 11840 , which was within the range of BHA (9679) and IR (15 848). The $N$ value of humin was 0.806 , which fell in between IR (0.732) and lipid (0.993). However, a precise comparison cannot be made according to the $K_{\mathrm{FOC}}$ values because of their different units as a result of nonlinearity. Therefore, the concentration-dependent organic carbon-normalized sorption coefficient $K_{\mathrm{OC}}$ at three selected concentration $\left(C_{\mathrm{e}}=0.005,0.05\right.$, and $\left.0.5 \mathrm{mg} / \mathrm{L}\right)$ were employed to compare the sorption capacity. Overall, $K_{\mathrm{OC}}$ was a concentration-dependent sorption coefficient, and lower $C_{\mathrm{e}}$ concentrations would result in higher $K_{\mathrm{OC}}$ values, as a result of nonlinear sorption. When $C_{\mathrm{e}}$ was at the higher concentrations $(0.5$ and $0.05 \mathrm{mg} / \mathrm{L})$, the $K_{\mathrm{OC}}$ followed the order lipid $>$ IR $>\mathrm{HA}>\mathrm{BHA}$. When $C_{\mathrm{e}}$ was at the lower concentration $(0.005 \mathrm{mg} / \mathrm{L})$, the $K_{\mathrm{OC}}$ of IR (68 764) was higher than that of lipid (54 088). Kang and Xing (7) progressively extracted HA and isolated humin into two fractions according to the different organic carbon contents. The sorption of phenanthrene by humic acids showed that the $K_{\mathrm{OC}}\left(C_{\mathrm{e}}=\right.$ $0.05 \mathrm{mg} / \mathrm{L}$ ) of the last extracted humic acid was 1.95 times that of the first extracted one, while the sorbed phenanthrene by low organic carbon content humin was 2.45 times that of the high organic carbon content humin, thereby indicating the heterogeneity of SOM for phenanthrene sorption. In the current study, the $K_{\mathrm{OC}}\left(C_{\mathrm{e}}=0.05 \mathrm{mg} / \mathrm{L}\right)$ of lipid was 3.7 times that of BHA, demonstrating the greater heterogeneous properties of humin. Weber et al. (1) suggested that $N$ value could be taken as an index of site energy distribution. Smaller $N$ values represent more heterogeneous sorption sites in nature. Thus, the lipid fraction with the largest $N$ value may have the least heterogeneous structure or composition, which was also demonstrated by its relative uniform distribution of alkyl carbon as revealed in the ${ }^{13} \mathrm{C}$ NMR spectra. The IR fraction with the smallest $N$ value may possess the most heterogeneous sorption sites.

IR fraction was described as the mineral-bound organic matter (14), as well as some plant and fungal residues in varying stages of decomposition, such as lignin and polysaccharides (26). Wang and Xing (27) found that aliphatic fractions in HA were preferentially adsorbed on both montmorillonite and kaolinite. Whereas, sorption of humic acid on clay minerals was reported to depend on the types of clay minerals and various solution conditions $(28,29)$. The humic acid fractions sorbed onto kaolinite are more aliphatic than those onto montmorillonite, while montmorillonite displays a higher uptake of aromatics. The relatively higher content of aromatic group in IR fraction implied the existence of lignin or lignin-like matter. Studies by Wang et al. (30) showed that the lignin's aliphatic $\mathrm{C}$ is about $43.2 \%$, suggesting a higher content of aromatic- $\mathrm{C}$ in lignin. The relatively lower content of aliphatic C in IR fraction $(7.1 \%)$ might be due to the extraction of lignin's polar and nonpolar aliphatic moieties by $\mathrm{NaOH}$ and MIBK in the fractionation procedure. The mineral-bound organic matter and lignin were reported as the important sources of nonlinear sorption of HOCs $(27,29$, 30 ). The $N$ values for kaolinite and montmorillonite clay bound humic acids were reported to be 0.72 and 0.75 , and the $K_{\mathrm{FOC}}$ values were 6329 and 7072 under $0.01 \mathrm{M} \mathrm{CaCl}_{2}$ and pH 4 conditions, respectively (29). The $N$ values for lignin were reported to be in the range of $0.801-0.956$, while the


our results.

Lipids, operationally defined as organic geochemicals that are soluble in nonpolar organic, are thought to be difficult to degrade microbiologically (31). Lipids can not only serve as a diagnostic index of soil biological activity, but also act as the dominant sorption domains in forest and agricultural soils $(32,33)$. Linear sorption was found for phenanthene on lipid-modified clay (34). Removal of the lipids from soils resulted in the increase of the nonlinear character of the 

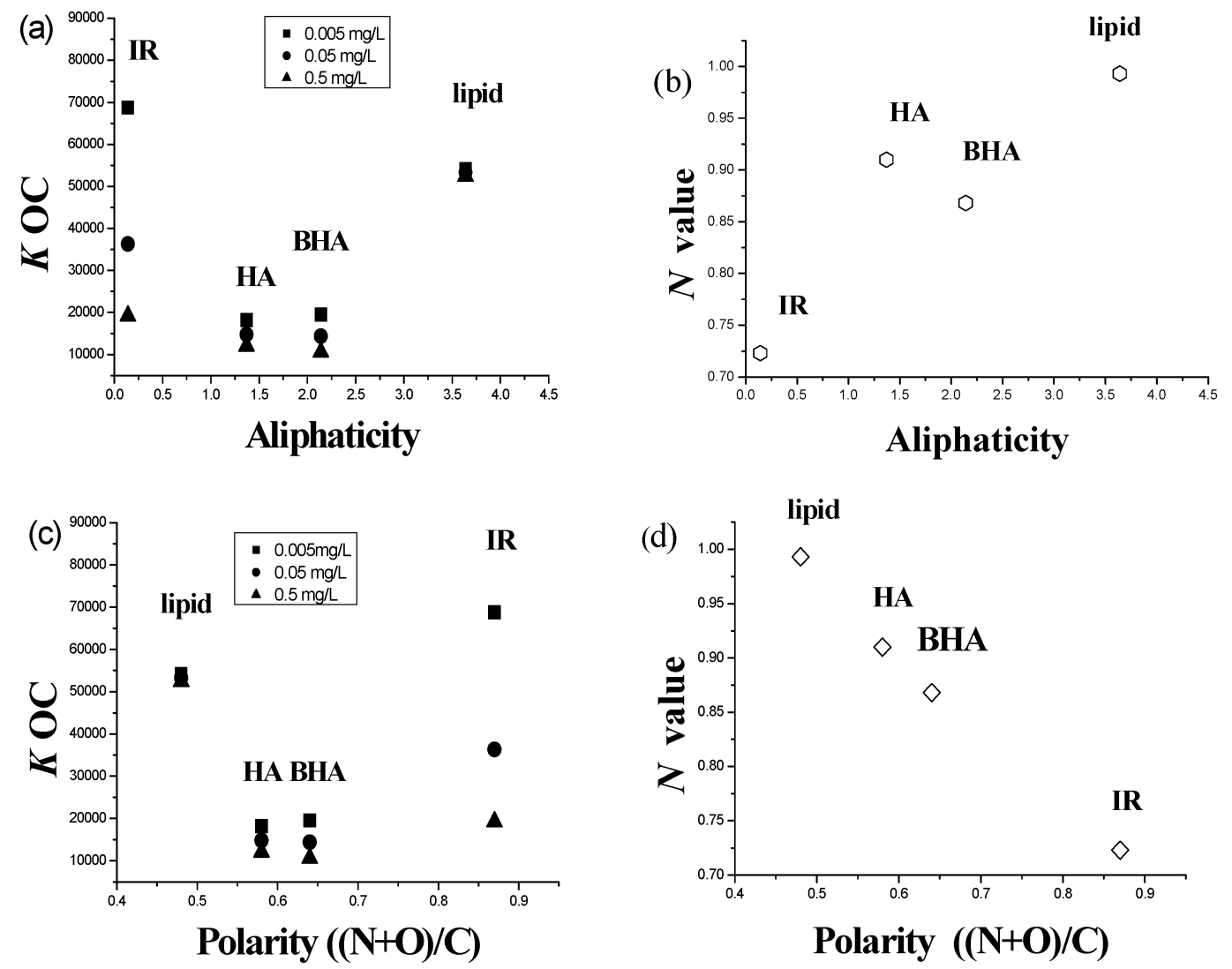

FIGURE 3. Relationship between the aliphaticity of HA, BHA, lipid, and IR fractions and the $K_{0 \mathrm{cc}}(\mathrm{a})$ and $\boldsymbol{N}$ values (b) of phenanthrene sorption; Relationship between the polarity of HA, BHA, lipid, and IR fractions and the Koc (c) and $\boldsymbol{N}$ values (d) of phenanthrene sorption.

sorption isotherms thereby indicating the linear nature of lipid sorbent (35). This evidence showed that the sorption of HOCs on lipid is mainly through partitioning.

Numerous studies dealing with the sorption of phenanthrene on HA extracted from various sources revealed the sorption nonlinearity $(7,8,22,27,36,37)$. Freundlich equation parameters $K_{\mathrm{FOC}}$ and $N$ of phenanthrene sorption were reported to be $11750-17982$ and $0.902-0.961$, respectively, for HA extracted from Canadian soils from different depth (36); 6224-30 277 and 0.9516-1.1514, respectively for HA from four Chinese soils (37); 8400-19 400 and 0.902-0.961, respectively for HA from American soils (7); and $51000-$ 51400 and $0.90-0.92$, respectively for HA from sediments (8). For the HA extracted from peat soil, the Freundlich equation parameters $K_{\mathrm{FOC}}$ and $N$ of phenanthrene sorption were 13056 and 0.874 , respectively (27). In our study the value of $K_{\mathrm{FOC}}$ was found to be 11298 , which is lower than those of sediment, but was within the range of soils and peat soil.

To the best of our knowledge, the sorption of HOCs on the BHA fraction had never been reported. Xie et al. (14) deemed that the BHA fraction is not merely inextracted humic acid because its ${ }^{13} \mathrm{C}$ NMR spectrum was significantly different from that of HA extracted from the same source. Kang and Xing (7) showed that compared with the former extracted humic acids, the later extracted humic acids are more aliphatic and less polar. Our results showed that the BHA is more aliphatic and polar than HA, thereby indicating the unique feature of BHA. However, its Freundlich equation parameters for phenanthrene sorption, $N$ and $K_{\mathrm{FOC}}$, were within the reported range of HA.

Effects of Fractionated SOM Properties on Freundlich Sorption Equation Parameters. Application of ${ }^{13} \mathrm{C}$ NMR spectra analyses facilitates the exploration of the relationship between the structural characteristics and Freundlich iso- therm sorption parameters, $K_{\mathrm{OC}}$ and $N(2)$. Nevertheless, it is still controversial whether aromatic or aliphatic groups are dominantly responsible for sorption of nonpolar aromatic sorbates. Based on the concept of "like dissolves like", it has often been assumed that the aromatic rather than the aliphatic domains of SOM will determine the sorption behavior of HOCs. The positive relation between $K_{\mathrm{OC}}$ values of phenanthrene and aromatic carbon content of the sorbents (37), and the sorption mechanism proposed as the overlap of $\pi$-electrons in the aromatic black carbon structure and the planar aromatic ring of PAH molecules (38) suggest the importance of aromatic carbons. However, the finding of greater phenanthrene sorption capacity in aliphatic biopolymers equivalent to those of aromatic carbons, and the positive correlation between phenanthrene $K_{\mathrm{OC}}$ values and alkyl-C contents undermined the role of aromatic carbon $(24,34)$. In our study it appears that there was no correlation between $K_{\text {OC }}$ values and aliphaticity of the SOM fractions (Figure 3a). Both the aliphatic-rich lipid and the aromatic-rich IR fractions showed relatively higher $K_{\mathrm{OC}}$ values, revealing the importance of both aromatic and aliphatic groups on the $K_{\mathrm{OC}}$. With the aliphaticity of SOM fractions increased, the $N$ values increased (Figure $3 b$ ) despite of the insignificant correlation between $N$ values and aliphatic components $(p>0.1)$. If we did not consider the data of HA that do not belong to the humin fraction, we could find the significant inverse correlation between the aliphaticity and the $N$ values $\left(R^{2}=0.998, p<\right.$ 0.05 ), which indicate the importance of aromatic carbon for isotherm nonlinearity, as shown by Xing (36).

In addition to the aromatic or aliphatic carbon contents, the effect of polarity on the sorption capacity and nonlinearity should also be considered. No correlation existed between $K_{\mathrm{OC}}$ and polarity of the SOM fractions (Figure $3 \mathrm{c}$ ), which is different from the reported data in the literatures $(7,20,23$, 25). Negative correlation was found between the polarity 
and the $N$ values of these four SOM fractions, with $R^{2}$ of 0.995, $p<0.05$ (Figure 3d), which is not in line with the results of Liang et al. (22). However, these observations are consistent with the results of Kang and Xing (7) although no regression analysis was carried out by these authors.

In conclusion, our results demonstrate the highly heterogeneous nature of SOM, particularly the BHA, lipid, and IR fractions of humin, even though all these fractions were extracted from a single peat soil. No correlation of $K_{\mathrm{OC}}$ with either aromatic or aliphatic carbon contents was observed. Both aliphatic-rich lipid and aromatic-rich IR fractions showed greater sorption capacity for nonpolar organic pollutants, which appears to be an important consideration in reevaluating the role of aliphatic and aromatic organic matter in the sorption of HOCs. All the SOM fractions showed the nonlinear sorption of phenanthrene except for lipid fraction. The negative relationship between polarity and the sorption nonlinearity ( $N$ values) and the positive relationship between the aliphaticity of BHA, lipid, and IR fractions and $N$ values revealed the important roles of SOM polarity and aromaticity in sorption nonlinearity.

\section{Acknowledgments}

This work was funded by the National Natural Science Foundation of China (grant nos. 20377048 and 20237010).

\section{Supporting Information Available}

The details for the isolation of humic acid (HA) and humin fractions procedure, determination of elemental composition and E4/E6 ratios of SOM fractions (Table S1), structural carbon distributions as obtained from ${ }^{13} \mathrm{C}$ NMR (Table S2), sequence of MIBK method (Figure S1), phenanthrene sorption kinetics on SOM fractions (Figure S2), relationship between the polarity index $((\mathrm{O}+\mathrm{N}) / \mathrm{C})$ and the percentage of polar organic carbon of SOM fractions (Figure S3), relationship between the polarity index $((\mathrm{O}+\mathrm{N}) / \mathrm{C})$ and the percentage of alkyl carbon of SOM fractions (Figure S4), sorption isotherms of phenanthrene by SOM fractions (Figure S5). This material is available free of charge via the Internet at http://pubs.acs.org.

\section{Literature Cited}

(1) Weber, W. J. Jr.; McGinley, P. M.; Katz, L. E. A distributed reactivity model for sorption by soils and sediments. 1. Conceptual basis and equilibrium assessments. Environ. Sci. Technol. 1992, 26, $1955-1962$.

(2) Huang, W.; Weber, W. J., Jr. A distributed reactivity model for sorption by soils and sediments. 10: Relationships between desorption, hysteresis, and the chemical characteristics of organic domains. Environ. Sci. Technol. 1997, 31, 2562-2569.

(3) Huang, W.; Peng, P.; Yu, Z.; Fu, J. Effects of organic matter heterogeneity on sorption and desorption of organic contaminants by soils and sediments. Appl. Geochem. 2003, 18, 955972.

(4) Xing, B.; Pignatello, J. J. Dual-mode sorption of low-polarity compounds in glassy poly (vinyl chloride) and soil organic matter. Environ. Sci. Technol. 1997, 31, 792-799.

(5) Cornelissen, G.; Gustafsson, Ö.; Bucheli, T. D.; Jonker, M. T. O.; Koelmans, A. A.; Van Noort, P. C. M. Extensive sorption of organic compounds to black carbon, coal, and kerogen in sediments and soils: Mechanisms and consequences for distribution, bioaccumulation, and biodegradation. Environ. Sci. Technol. 2005, 39, 6881-6895.

(6) Leboeuf, E. J.; Weber, W. J. Jr. A distributed reactivity model for sorption by soils and sediments. 8. Sorbent organic domains: discovery of a humic acid glass transition and an argument for a polymer-based model. Environ. Sci. Technol. 1997, 31, 16971702.

(7) Kang, S.; Xing, B. Phenanthrene sorption to sequentially extracted soil humic acids and humins. Environ. Sci. Technol. 2005, 39, 134-140.

(8) Oren, A.; Chefetz, B. Sorption-desorptin behavior of polycyclic aromatic hydrocarbons in upstream and downstream river sediments. Chemosphere 2005, 61, 19-29.
(9) Pan, B.; Xing, B. S.; Liu, W. X; Tao, S.; Lin, X. M; Zhang, X. M.; Xiao, Y.; Dai, H. C.; Yuan, H. S. Distribution of sorbed phenanthrene and pyrene in different humic fractions of soils and importance of humin. Environ. Pollut. 2006, 143, 2433.

(10) Rice, J. A.; MacCarthy, P. Isolation of humin by liquid-liquid partitioning. Sci. Total Environ. 1989, 81/82, 61-69.

(11) Doick, K. J.; Burauel, P.; Jones, K. C.; Semple, K. T. Distribution of aged ${ }^{14} \mathrm{C}$-PCB and ${ }^{14} \mathrm{C}$-PAH residues in particle-size and humic fractions of an agricultural soil. Environ. Sci. Technol. 2005, 39, $6575-6583$.

(12) Kohl, S. D.; Rice, J. A. The binding of contaminants to humin: A mass balance. Chemosphere 1998, 36, 251-261.

(13) Rice, J. A.; MacCarthy, P. Composition of humin in stream sediments and peat. In Organic Substances and Sediments in Water, Part 1. Humics and Soils; Baker, R. A., Ed.; Lewis Publishers: Chelsea, MI, 1991.

(14) Xie, H.; Guetzloff, T. F.; Rice, J. A. Fractionation of pesticide residues bound to humin. Soil Sci. 1997, 162, 421-429.

(15) Rice, J. A. Humin. Soil Sci. 2001, 166, 848-857.

(16) Chiou, C. T.; McGroddy, S. E.; Kile, D. E. Partition characteristics of polycyclic aromatic hydrocarbons on soils and sediments. Environ. Sci. Technol. 1998, 32, 264-268.

(17) Wauchope, R. D.; Koskinen, W. C. Adsorption-desorptin equilibria of herbicides in soil: A thermodynamic perspective. Weed Sci. 1983, 31, 504-512.

(18) Nelson, D. W.; Sommers, L. E. In Methods of Soil Analysis, Part 2. Chemical and Microbiological properties, Agronomy Monograph 9; 2nd ed.; Page, A. L, Miller, R., Keeney, D. R., Eds.; ASA and SSSA: Madison, WI, 1982.

(19) Senesi, N.; D'Orazio, V.; Ricca, G. Humic acids in the first generation of Eurosoils. Geoderma 2003, 116, 325-344.

(20) Chen, B.; Johnson, E. J.; Chefetz, B.; Zhu, L.; Xing, B. Sorption of polar and nonpolar aromatic organic contaminants by plant cuticular materials: Role of polarity and accessibility. Environ. Sci. Technol. 2005, 39, 6138-6146.

(21) Shen, Q.; Zhong, L. Lignin-based carbon films and controllable pore size and properties. Mater. Sci. Eng. A 2006, 445-446, 731735.

(22) Liang, C.; Dang, Z.; Xiao, B.; Huang, W.; Liu, C. Equilibrium sorption of phenanthrene by soil humic acids. Chemosphere 2006, 63, 1961-1968.

(23) Rutherford, D. W.; Chiou, C. T.; Kile, D. E. Influence of soil organic matter composition on the partition of organic compounds. Environ. Sci. Technol. 1992, 26, 336-340.

(24) Mao, J. D.; Hundal, L. S.; Thompson, M. L.; Schmidt-Rohr, K. Correlation of poly(methylene)-rich amorphous aliphatic domains in humic substances with sorption of a nonpolar organic contaminant, phenanthrene. Environ. Sci. Technol. 2002, 36, 929-936.

(25) Xing, B.; McGill, W. B.; Dudas, M. J. Cross-correlation of polarity curves to predict partition coefficients of nonionic organic contaminants. Environ. Sci. Technol. 1994, 28, 1929-1933.

(26) Halcher, P. G.; Breger, G. E.; Maciel, I. A.; Szeverenyi, N. M. In Humic Substances in Soil, Sediment and Water; Geochemistry, Isolation and Characterisation; Aiken, G. R., McKenight, D. M., Wershaw, R. L., MacCarty, P., Eds.; John Wiley \& Sons: New York, 1985.

(27) Wang, K.; Xing, B. Structural and sorption characteristics of adsorbed humic acid on clay minerals. J. Environ. Qual. 2005, $34,342-349$.

(28) Feng, X.; Simpson, A. J.; Simpson, M. J. Chemical and mineralogical controls on humic acid sorption to clay mineral surface. Org. Geochem. 2005, 36, 1553-1566.

(29) Feng, X.; Simpson, A. J.; Simpson, M. J. Investigating the role of mineral-bound humic acid in phenanthrene sorption. Environ. Sci. Technol. 2006, 40, 3260-3266.

(30) Wang, X.; Cook, R.; Tao, S.; Xing, B. Sorption of organic contaminants by biopolymers: role of polarity, structure and domain spatial arrangement. Chemosphere 2006, 66, 14761484.

(31) Jandl, G.; Leinweber, P.; Schulten, H.; Ekschmitt, K. Contribution of primary organic matter to the fatty acid pool in agricultural soils. Soil Biol. Biochem. 2005, 37, 1033-1041.

(32) Almendros, G.; Guadalix, M. E.; Gonzalez-Vila, F.; J. Martin, F. Preservation of aliphatic macromolecules in soil humins. Org. Geochem. 1996, 24, 651-659.

(33) Salloum, M. J.; Chefetz, B.; Hatcher, P. G. Phenanthrene sorption by aliphatic-rich natural organic matter. Environ. Sci. Technol. 2002, 36, 1953-1958. 
(34) Chen, B.; Xing, B. Sorption and conformational characteristics of reconstituted plant cuticular waxes on montmorillonite. Environ. Sci. Technol. 2005, 39, 8315-8323.

(35) Kohl, S. D.; Rice, J. A. Contribution of lipids to the nonlinear sorption of polycyclic aromatic hydrocarbons to soil organic matter. Org. Geochem. 1999, 30, 929-936.

(36) Xing, B. Sorption of naphthalene and phenanthrene by soil humic acids. Environ. Pollut. 2001, 111, 303-309.

(37) Xu, D.; Zhu, S.; Chen, H.; Li, F. Structural characterization of humic acids isolated from typical soils in China and their adsorption characteristics to phenanthrene. Colloid Surf. A 2006, $276,1-7$.
(38) Zhu, D. Q.; Hyun, S.; Pignatello, J. J.; Lee, L. S. Evidence for $\pi-\pi$ electron donor-acceptor interactions between $\pi$-donor aromatic compounds and $\pi$-acceptor sites in soil organic matter through pH effect on sorption. Environ. Sci. Technol. 2004, 38, 43614368.

Received for review September 22, 2006. Revised manuscript received January 11, 2007. Accepted February 5, 2007.

ES062262S 